\title{
BSM search with high intensity muon beam in MEG II experiment
}

\section{Kei leki*University of Tokyo}

E-mail: iekikei@icepp.s.u-tokyo.ac.jp

Flavor violating decay of muon is a good probe of new physics beyond SM. Many well motivated new physics models predict $\mu \rightarrow e \gamma$ decay to occur at large branching ratio (e.g. $\mathscr{O}\left(10^{-14}\right)$ in SUSY-seesaw). MEG II experiment will search for $\mu \rightarrow e \gamma$ decay with target sensitivity down to $6 \times 10^{-14}$, which is an order of magnitude better than the sensitivity of MEG. MEG II experiment utilizes world's most intense DC muon beam at Paul Scherrer Institute. All of the detectors are upgraded from MEG to cope with increased rate of accidental backgrounds. In 2018, preengineering run was performed with all the upgraded detectors installed for the first time. Status and prospect of MEG II will be presented.

European Physical Society Conference on High Energy Physics - EPS-HEP2019 -

10-17 July, 2019

Ghent, Belgium

${ }^{*}$ Speaker. 


\section{Motivation to search $\mu \rightarrow e \gamma$}

Flavor violation is already discovered in quark sector and neutrino sector, but not in charged lepton sector. Charged lepton flavor violation (cLFV) is not expected to occur in the framework of Standard Model, but it is predicted to occur in many well-motivated beyond Standard Model theories, because there is no fundamental rule to prohibit cLFV. Therefore, discovery of cLFV would be a smoking gun signature of new physics.

MEG II experiment searches for LFV muon decay, $\mu \rightarrow e \gamma$. This decay can occur if neutrino oscillation is taken into account (Fig. 11 left), but the branching ratio is expected to be negligibly small $\left(\sim 10^{-54}\right)$ due to small neutrino masses. On the other hand, with contributions from new physics particles via loop diagrams (Fig. 1 1 right), the branching ratio of this decay becomes as large as $\mathscr{O}\left(10^{-14}\right)$. Current upper limit on branching ratio is $4.2 \times 10^{-13}$ (90\% C.L., MEG experiment), which is already in a region predicted by new physics models. Figure 2 shows an example of parameter space in SUSY-seesaw, with contours describing the region excluded by the MEG experiment. MEG II experiment will search for $\mu \rightarrow e \gamma$ with target sensitivity further improved down to $6 \times 10^{-14}$.

Following sections describe the final result of MEG and the upgrade concept of MEG II. Status of the upgrade is also described. More details of the MEG II experiment is available in [2].
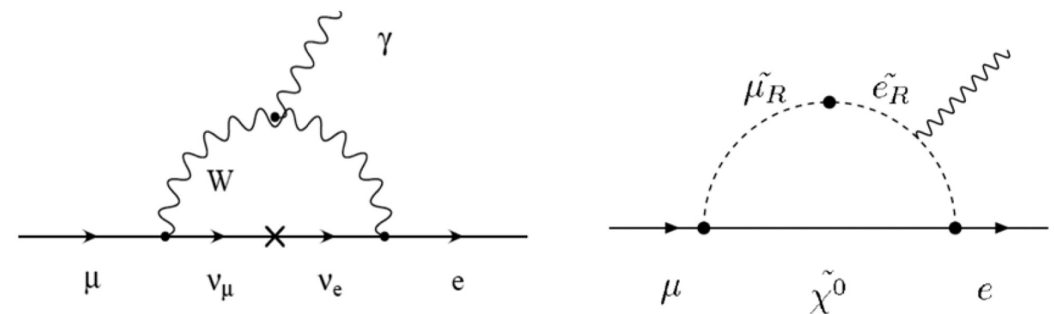

Figure 1: Feynman diagram of $\mu \rightarrow e \gamma$ decay through neutrino oscillation (left) and through new physics particle (right).

\section{Previous search in MEG}

$\mu \rightarrow e \gamma$ decay can be identified by detecting $e^{+}$and $\gamma$ emitted in back-to-back at the same timing, with same energy $\left(E_{e}=E_{\gamma}=52.8 \mathrm{MeV}\right)$. On the other hand, dominant background is an accidental coincidence of $e^{+}$and $\gamma$. The rate of this background is proportional to the square of the muon beam rate. In order to distinguish this background from $\mu \rightarrow e \gamma$, it is important to measure the energy, timing and direction of $e^{+}$and $\gamma$ with good precision.

The MEG experiment took place at Paul Scherrer Institute (PSI) in Switzerland. Main characteristics of MEG are as follows:

- High intensity continuous muon beam at PSI $\left(3 \times 10^{7} \mu / \mathrm{sec}\right)$

- High resolution detectors for $\gamma$ and $e^{+}$ 


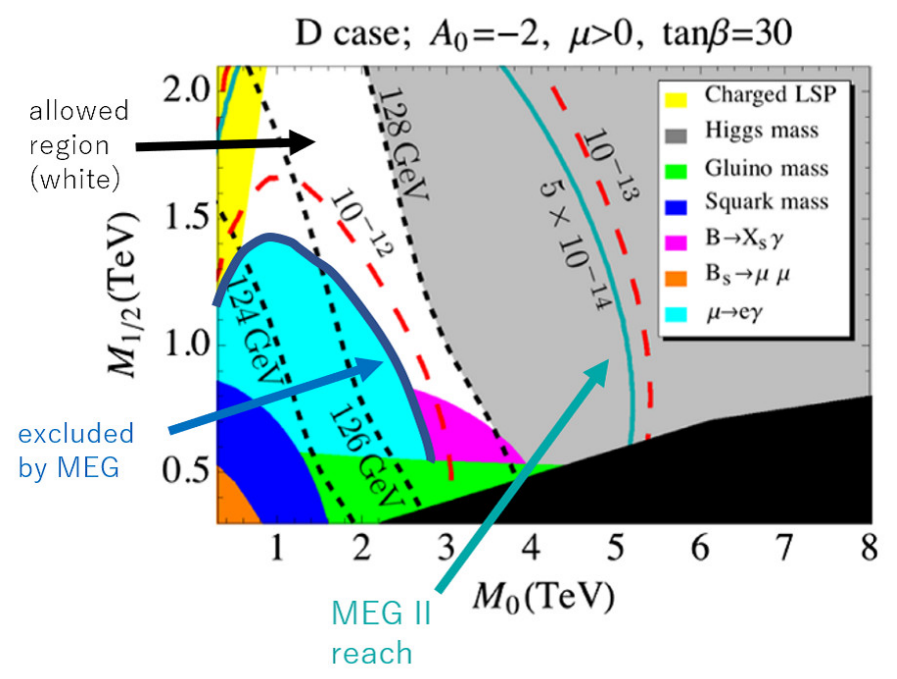

Figure 2: Example of parameter space excluded by $\mu \rightarrow e \gamma$ search (original plot from [1]).

PSI proton cyclotron provides world's most high intensity continuous muon beam. So called "surface beam" (muons from decay of pions stopping near the surface of proton target) is used. Momentum spectrum of such muons have sharp peak around $29 \mathrm{MeV} / \mathrm{c}$, and therefore it can be stopped by thin stopping target with high efficiency.

MEG took muon beam data from 2008 to 2013. Total amount of muons stopped on target was $7.5 \times 10^{14}$. Physics analysis was performed based on the measurement of energy $\left(E_{e}, E_{\gamma}\right)$, timing difference $\left(t_{e \gamma}\right)$ and opening angle $\left(\theta_{e \gamma}\right)$ of $e^{+}$and $\gamma$. Since there were no excess of signal events observed (Fig. 3), upper limit of $\operatorname{Br}(\mu \rightarrow e \gamma)<4.2 \times 10^{-13}$ (90\% C.L.) was obtained. This is 30 times stronger limit than the previous experiment, and gives strong constraint on new physics models.
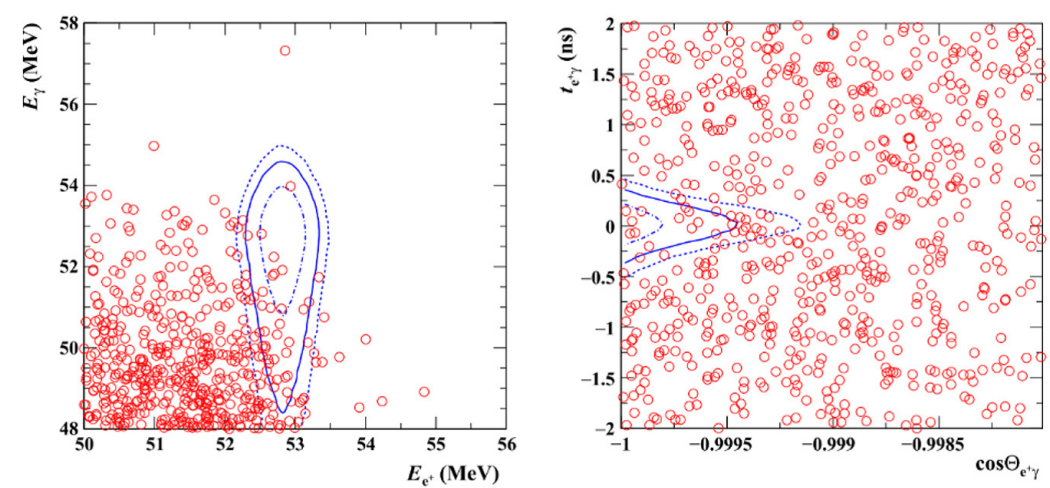

Figure 3: $E_{e}, E_{\gamma}, t_{e \gamma}$ and $\theta_{e \gamma}$ distributions of events observed in MEG. No significant excess is observed in the contours where we expect to see $\mu \rightarrow e \gamma$ events.

\section{MEG II upgrade}

MEG II experiment aims to search $\mu \rightarrow e \gamma$ with sensitivity improved from MEG by one order 
of magnitude $\left(6 \times 10^{-14}\right)$. Main subjects of the upgrade project is as follows:

- Increase the muon beam intensity by more than factor of two $\left(7 \times 10^{7}\right)$.

- Improve all detector resolutions (energy, position and time) by factor of two. Also improve the detection efficiency ( of $e^{+}$) by factor of two.

Muon beam with higher intensity was already available during MEG data taking, but the rate was reduced in MEG because otherwise the rate of accidental background was too high. With improved energy resolution in MEG II, it is possible to improve the sensitivity without being limited by accidental backgrounds.

Figure 4 shows the overview of MEG II detector apparatus. Both $e^{+}$and $\gamma$ detector are upgraded. In addition, there is a new detector to identify background events.

Positron detector consists of drift chamber (track measurement) and timing counter (time measurement). Both of these are placed inside gradient magnetic field, produced by a magnet reused from MEG. Gradient magnetic field bends low momentum positron tracks so that it does not hit the drift chamber, while keeping high efficiency for signal positron.

Liquid xenon (LXe) detector measures the gamma-ray. The cryostat and $900 \mathrm{LXe}$ are reused from MEG, but the photon sensors are replaced with compact SiPMs to improve the resolutions.

Following sections describe each components of the detectors.

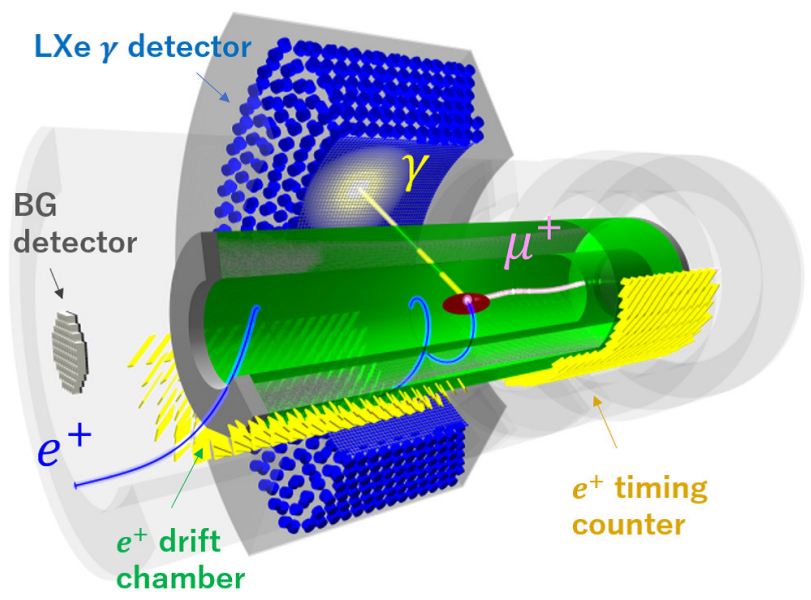

Figure 4: MEG II detector overview.

\section{1 $e^{+}$drift chamber}

Single volume cylindrical drift chamber is developed for measurement of $e^{+}$trajectories 5 , In MEG, efficiency for detecting $e^{+}$was lost by scattering of $e^{+}$at the edge of drift chamber modules before the track reached timing counter. With a single volume chamber, efficiency is expected to improve by a factor of 2 , because there is less amount of materials between drift chamber and timing counter. 


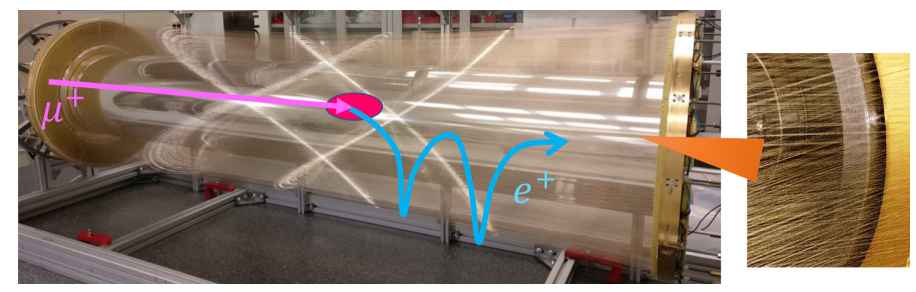

Figure 5: Positron drift chamber.

As in MEG, the amount of materials is minimized so that multiple scattering is minimized. For this reason, helium based light gas ( $90 \% \mathrm{He}, 10 \%$ iso-butane) and extremely thin wires ( $20 \mu \mathrm{m}$ Tungsten for signal, 40-50 $\mu \mathrm{m}$ Aluminum for field wires) are selected. Total amount of materials along $e^{+}$trajectory is $1.6 \times 10^{-3}$ radiation length, which is $20 \%$ smaller than that in MEG.

Wires are arranged to form 9 concentric layers. Each layer contains sense wires with alternating signs of stereo angles $\left(6.0-8.5^{\circ}\right)$ for reconstruction of longitudinal hit position. Sense wires are surrounded by field wires to form drift cells. High granularity of wires (6.6-9.0 mm drift cells) allows to reconstruct $e^{+}$tracks with good resolution in high rate environment. The expected energy resolution and angle resolution are $\sim 130 \mathrm{keV}$ and $5 \mathrm{mrad}$, respectively.

\section{$3.2 e^{+}$timing counter}

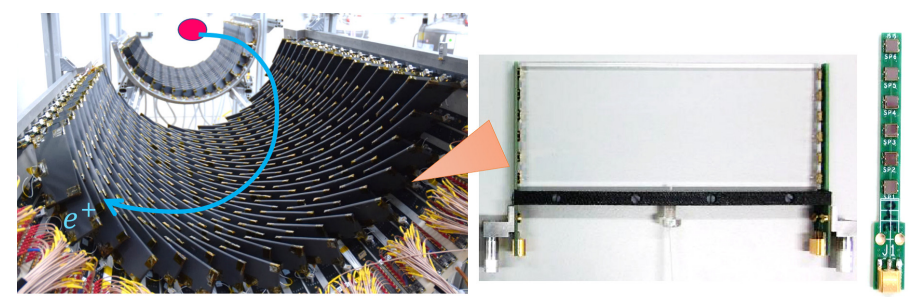

Figure 6: Positron timing counter.

Positron timing is measured by timing counter, which consists of 512 plastic scintillator counters (Fig. 6). Thanks to high granularity, each $e^{+}$hits 9 counters in average. Multiple time measurement with multiple counters improves the time resolution. Each counter is composed of fast plastic scintillator (BC-422 from Saint-Gobain) with 6 silicon photomultipliers (SiPMs) connected on each sides. Six SiPMs are connected in series and read out as one channel. Time resolution of $<40 \mathrm{ps}$ is already achieved in the commissioning run with muon beam (Fig. 7).

\subsection{LXe gamma detector}

Liquid Xenon (LXe) detector measures the energy, time and position of gamma. LXe is ideal material for the measurement of gamma with good resolution and high efficiency. It has high light yield ( $\sim 75 \%$ of $\mathrm{NaI})$, short radiation length $(\sim 2.8 \mathrm{~cm})$ and fast response $(\sim 45 \mathrm{~ns}$ decay time for $\gamma)$. In MEG II, we replaced 216 2-inch PMTs at gamma entrance face to $409612 \mathrm{~mm}$ square SiPMs (Fig. 8).

These SiPMs are sensitive to vacuum ultraviolet scintillation light from Xe. It has been newly developed in collaboration with Hamatsu Photonics. In MEG, energy resolution was worsened by 


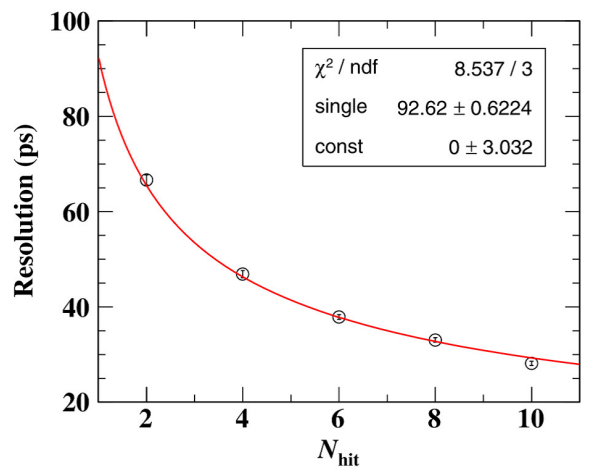

Figure 7: Timing resolution of timing counter, measured by 'odd-even' analysis.


Figure 8: Liquid xenon gamma-ray detector.

non-uniformity of the sensor coverage because of gaps between PMTs. With SiPMs uniformly covering the gamma entrance face, energy resolution is expected to improve from $\sim 2 \%$ (MEG) to $\sim 1 \%$ (MEG II). Thanks to improved granularity, position resolution is also expected to improve by a factor of two.

Commissioning of detector was successfully done with background gamma in high intensity muon beam and also with $17.6 \mathrm{MeV}$ monochromatic gamma from ${ }_{3}^{7} \mathrm{Li}(p, \gamma){ }_{4}^{8} \mathrm{Be}$. Right side of figure 8 shows an example of $\gamma$ event detected in commissioning runs.

\subsection{Background identifying detector}

Background identifying detector is a detector newly added in MEG II. This detector identifies radiative decay of muons ( $\mu \rightarrow e v v \gamma$ ), which is a main source of background gamma. This radiative decay can be identified by detecting low energy $e^{+}$which is emitted at the same time. Such $e^{+}$drifts in magnetic field towards upstream or downstream of the beam, so it can be detected by a detector placed in beam line (Fig. (9).

The detector is composed of 12 plastic scintillator plates and 76 LYSO crystals. Plastic scintillator measures the timing of $e^{+}$to identify radiative decay by time coincidence of $\gamma$ and $e^{+}$. LYSO crystals measures the energy of $e^{+}$to distinguish $e^{+}$from radiative decay with $e^{+}$from Michel decay $(\mu \rightarrow e v v)$, which tends to have higher energy. Segmented design was necessary for operation 

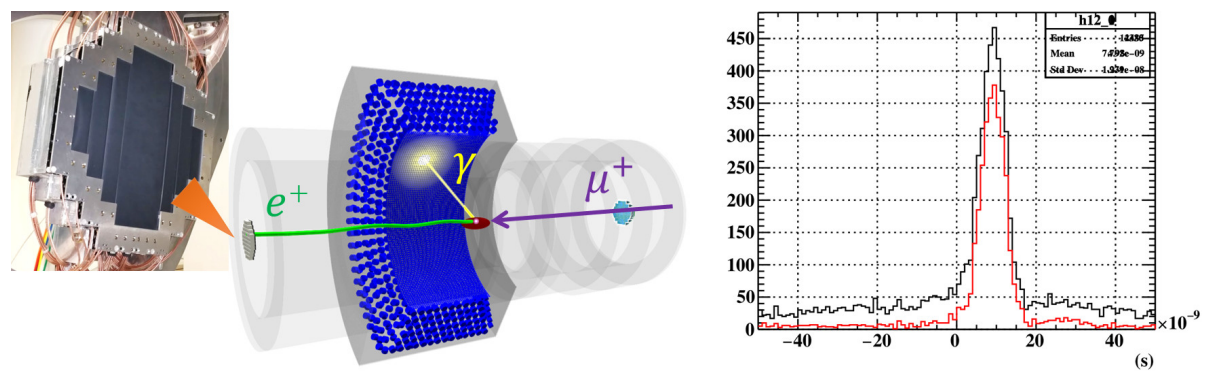

Figure 9: Background identifying detector $\operatorname{RDC}($ left $)$ and $\operatorname{LXe}(\gamma)-\operatorname{RDC}\left(e^{+}\right)$timing difference distribution (right).

in high rate beam. SiPMs are used for readout of scintillation light as it is compact and insensitive to magnetic field.

\subsection{DAQ and trigger}

Readout electronics called WaveDAQ is newly developed by PSI and INFN. All of the detectors ( $\sim 9000$ channels in total) are read out with DRS (Domino Ring Sampler) chip which performs waveform digitization at 1-2 GHz. Integrated trigger system allows to have sophisticated trigger for $\mu \rightarrow e \gamma$ event with reduced rack space.

\section{Status and prospect}

In 2018, construction of the detectors was finished except for the readout electronics. Detector commissioning with muon beam and calibration sources is ongoing. All of the readout electronics is expected to be ready in 2020 for the full operation of the detectors. Detector commissioning will continue in 2020 , followed by physics data taking. Branching ratio sensitivity is expected to exceed the current upper limit of MEG within first few months, and it will reach $6 \times 10^{-14}$ after 3 years of operation.

\section{Summary}

$\mu \rightarrow e \gamma$ is a good probe of physics beyond standard model. There are many well-motivated new physics models (SUSY, extra dimension etc.) which predicts $\mu \rightarrow e \gamma$ branching ratio within the current experimental reach $\left(O\left(10^{-14}\right)\right)$. MEG II experiment utilizes world's most high intensity continuous muon beam at PSI and innovative new detector technologies to search $\mu \rightarrow e \gamma$ decay with target sensitivity improved from MEG down to $6 \times 10^{-14}$. All the detectors are installed and tested with part of the readout electronics. Commissioning with full electronics is planed in 2020, and the physics data taking is expected to start soon.

\section{References}

[1] T. Goto et. al., Lepton flavor violation in the supersymmetric seesaw model after the LHC 8 TeV run, Phys. Rev. D91 033007 (2015)

[2] A.M. Baldini et. al., The design of the MEG II experiment, Eur. Phys. J. C78 380 (2018) 